\title{
RESEARCH AND IMPLEMENTATION OF GEODETIC DATABASE SYSTEM
}

\author{
Yongshang Wang ${ }^{1}$, Peng Zhang ${ }^{1}$, Xiaoqing Wang ${ }^{1}$, Zhanyi Sun ${ }^{1}$ \\ ${ }^{1}$ National Geomatics Center of China, Beijing, China, ys@ngcc.cn
}

KEY WORDS: Geodetic Data, Database, System, Research, Construction

\begin{abstract}
:
With the gradual formation of the space technical system of National Geodetic Datum, the storage management and application services of the geodetic data have been developing rapidly. This paper studies Geodetic data content, characteristics, classification principles and classification methods in the geodetic technology system and initially forms a standard classification system of geodetic data. On the basis of studying the key technical links to the modern Geodetic Datum comprehensive data acquisition, storage, management and service, this paper has tackled the real-time data optimal storage based on DBFS technology. Technical problems such as multi-dimensional data integration and docking of the software, building a safe and standardized management and the geodetic data service system, coordinating the application of network resources, storage resources, computing resources and other soft and hard environment elements, achieving efficient management and service of land, sea and air integrated observation data results, greatly improving the management ability and application service level of Geodetic data, for surveying and mapping. Business operation of the benchmark database provides support and promotes the social application on geodetic results.
\end{abstract}

\section{INTRODUCTION}

The new geodetic technology system characterized with high precision, real-time and popularization has gradually formed in the wave of new technological revolution. Taking advantage of modern information technology, the new geodetic technology system has new features for technology sharing and data sharing, in which geodetic data and positioning applications and geodetic applications can meet the requirements of real-time, public-shared and interactivity.

Geodetic Data Standards are the cornerstone in process of geodetic information, which describes scientifically the geodetic data processing, management and service, as well as the theory, methods and procedures used for implementation. Along with the development of geodetic information, the width and depth of data standardization application will far exceed the level of traditional standardization. With the advancement of measurement technology, there are less technical constraints in the relatively simple instruments operation while more complicated data structure can be analyzed, and the more urgent socialization services requirements to be fit. The focus of geodetic standardization will shift from operational standards to data standards.

In this paper, the content, characteristics, classification principles and methods of geodetic data are studied for the new characteristics of modern geodetic information.

\section{THE FEATURES OF GEODETIC DATA}

Geodetic data is the basic data for national scientific research, national defense and economic construction. It has strong spatial characteristics. Geodetic control points as permanent stations have precise horizontal and vertical coordinates, which is the foundation of determining the location of other points. All of these data have the following features:

1) Accuracy: Geodetic data has high precision, with clean (no gross error) original observations and rigorous data processing model and complete and reliable description. Accuracy is the key to establish a geodetic database. Thus certain measures must be taken to ensure the correctness and reliability of the warehousing data.

2) Long-term and real-time coexistence: Geodetic control points are permanent buried, so all control point information is preserved and used for a long time. The current status of the points is required to be accurately presented, such as the integrity of the point, whether it is available, any changes, etc., which matters for real-time ability.

3) Periodicity and accumulation: The geodetic survey should be repeated in a certain period of time, such as the national leveling network re-measurement, which is cyclical. With the continuous development of research work and national economic construction, the geodetic work is expanding and data continues is accumulating.

4) Complex data processing procedure: A series of complex procedure are performed to process the geodetic data from the raw observation data preprocessing to adjustment, such as gross error detection, naturalization 
and projection, adjustment, quality inspection and accuracy assessment etc.

According to the characteristics of geodetic data mentioned above, each data entity in the database can be classified into four types (as shown in Figure 1): observation data class, result data class, summary data class and auxiliary data class.

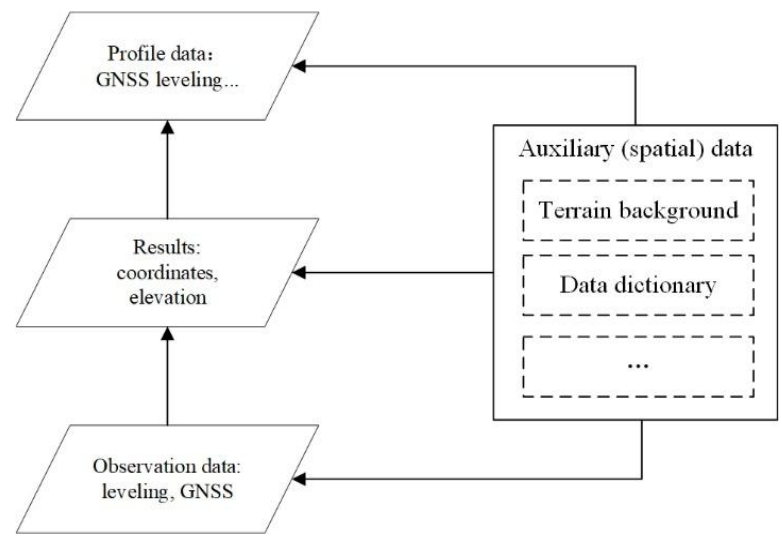

Figure 1, Data Entity in the database

The observed data is the original measurement record, including GNSS observation data, leveling observation data, and gravity observation data. These data are low-structured and often use file as the application granularity.

The result data is proceed professionally from the actual observation data, including GNSS coordinate results, elevation results, gravity results, time series, baseline accuracy and other result data. These results are highly structured and have fine application granularity.

The summary data describes the characteristics and summary information of GNSS points, level points, gravity points, mainly site information, point descriptions, and descriptive document files, which are less standardized.

The auxiliary data provides an electronic map, an administrative division, and a data dictionary (gives a database design) for better present of the spatial distribution of the summary data, the result data, and the observation data, and can be used as an auxiliary information data storage common to a plurality of types of databases.

\section{GEODETIC DATA CLASSIFICATION AND}

\section{DATA CONTENT}

The "National Geodetic Basic Technical Regulations" embodies four major categories of national geodetic surveys: geodetic and terrestrial control networks, elevation and elevation control networks, gravity and gravity measurement control networks, and depth datum. The "Basic Requirements for Geodetic Database" follows the classification of these four categories, and classify the data content of the geodetic database according to the different data characteristics and features of national, regional (provincial, municipal, county) and industrial geodetic survey.

Except the establishments of national datum and local datum, the complete control network is not necessary for other projects. So the classification is based on the type of results and measurement techniques instead of the control network. The first-level classification divides the data into datum, triangulation data, spatial positioning data, elevation measurement data, and gravity measurement data. The secondary classification is based on the content of results data. The data classification is shown in Figure 2.

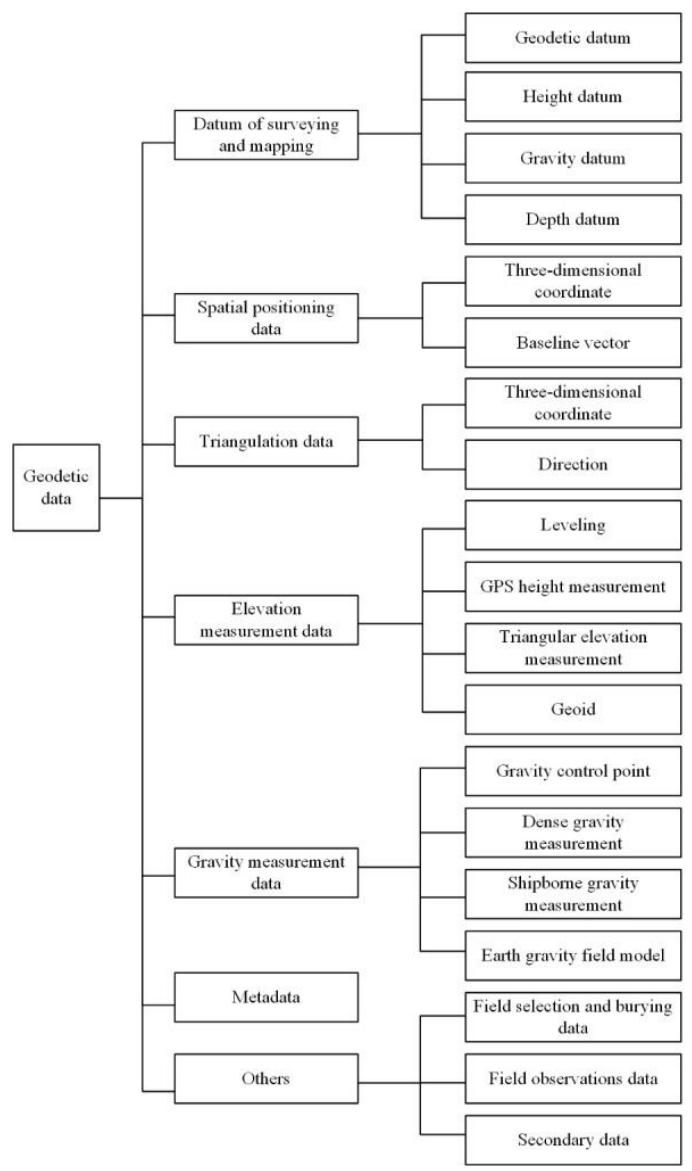

Figure 2, Data Classification

When setting the standards, the basic content of each data type is specified. The basic content is based on the results of internal data processing at first. Then the basic data content of each type of achievement data and metadata is clarified according to the needs of data building, system function development, and results usage. 


\section{CONSTRUCTION OF GEODETIC DATABASE BASED ON DATA STANDARDS}

The designed main process is shown in Figure 3, which presents a unified development of geodetic data management and application services. The functions of the data management platform are designed and arranged for the efficient operation of this main process.

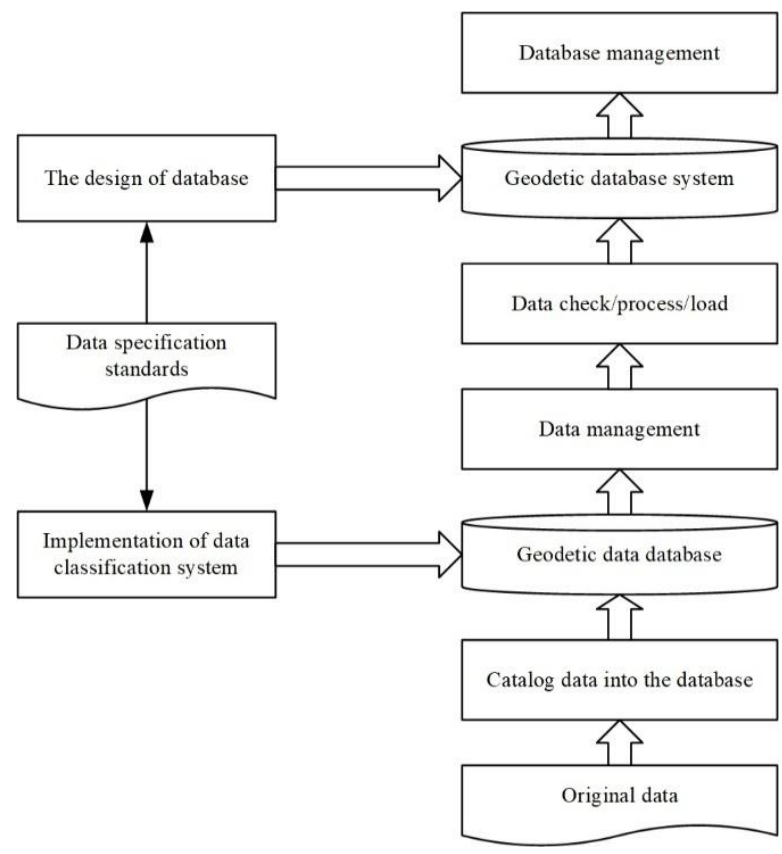

Figure 3, Geodetic Database Construction

A geodetic data database and a geodetic data framework consisting of multiple geodetic results database entities are provided. The geodetic data database is used to classified-centralized storage existing and future geodetic raw data. Each geodetic database is used to standardized storage of "standard" data based on data processing. Around the daily operation of geodetic data architecture, a data management environment with superior performance and complete functions is formed. The main process of data management platform consists of data cataloging and storage, data management, data inspection/processing/loading, results database management, and results database design.

\subsection{Geodetic database organization:}

Classification and organization are achieved according to the geodetic professional type and data content. The result data are stored by point or grid unit, which can reflect the logical relationship between the measurement network, the route and the point. A logical relationship should be established if there is a connection between different geodetic professional types and data content. Metadata is organized in the same way as the data objects described.

\subsection{Geodetic database storage requirements:}

The geodetic data stored in the warehouse should be accepted by a professional quality inspection agency, passed a series of professional data check procedure on correctness, integrity and logical relationship of the inbound data etc. Here is the general check list:

1) Check the correctness of the inbound data content and the correctness of the metadata;

2) check the integrity of the data items of various types of data and the amount of data in the warehouse;

3) Check the logical relationship between the measurement network, route, and point, and check the logical relationship between different measurement professional types and data content.

\subsection{Implementing efficient access to real-time data file data based on DBFS Technology.}

Generally, unstructured file data is stored and managed by both database system and file system, which makes up for the deficiency of file data access performance by sacrificing the advantages of database. The database ORACLE DBFS technology overcomes the disadvantage of the database management system in accessing binary LOB data. By improving the storage mechanism, the performance of LOB data access has reached the same level as file system, and even improved in some cases. At the same time, in order to support the access of file data by existing application systems, ORACLE DBFS technology not only realizes the mechanism of database application interface accessing LOB data, but also adds the file system application interface of LOB data. In modern surveying and mapping datum, there are a large number of unstructured file data types. Using DBFS technology with database file system-specific storage can take into account both database security and file access performance in order to meet the efficient utilization and application requirements of file data. In DBFS, a collection of PLSQL stored procedures provides basic operations for accessing file systems. The DBFS directory library allows each database user to create one or more file systems and can be used by clients. Each file system has a proprietary data table to ensure the content of the file system. ORACLE DBFS creates a standard file system structure on top of files and directories and stores data in database base tables. Real-time data access mechanism based on DBFS which showing in figure 3.

\section{THE ARCHITECTURE OF GEODETIC DATABASE SYSTEM}

The geodetic database consists three parts: geodetic data, management system and supporting environment, as shown in Figure 4. Among them, geodetic data is the core of the geodetic database, which is divided into geodetic control network data, elevation control network data, gravity control network data and depth reference data; Management system and supporting environment are software, hardware and network conditions for data storage, management, operation and maintenance. 


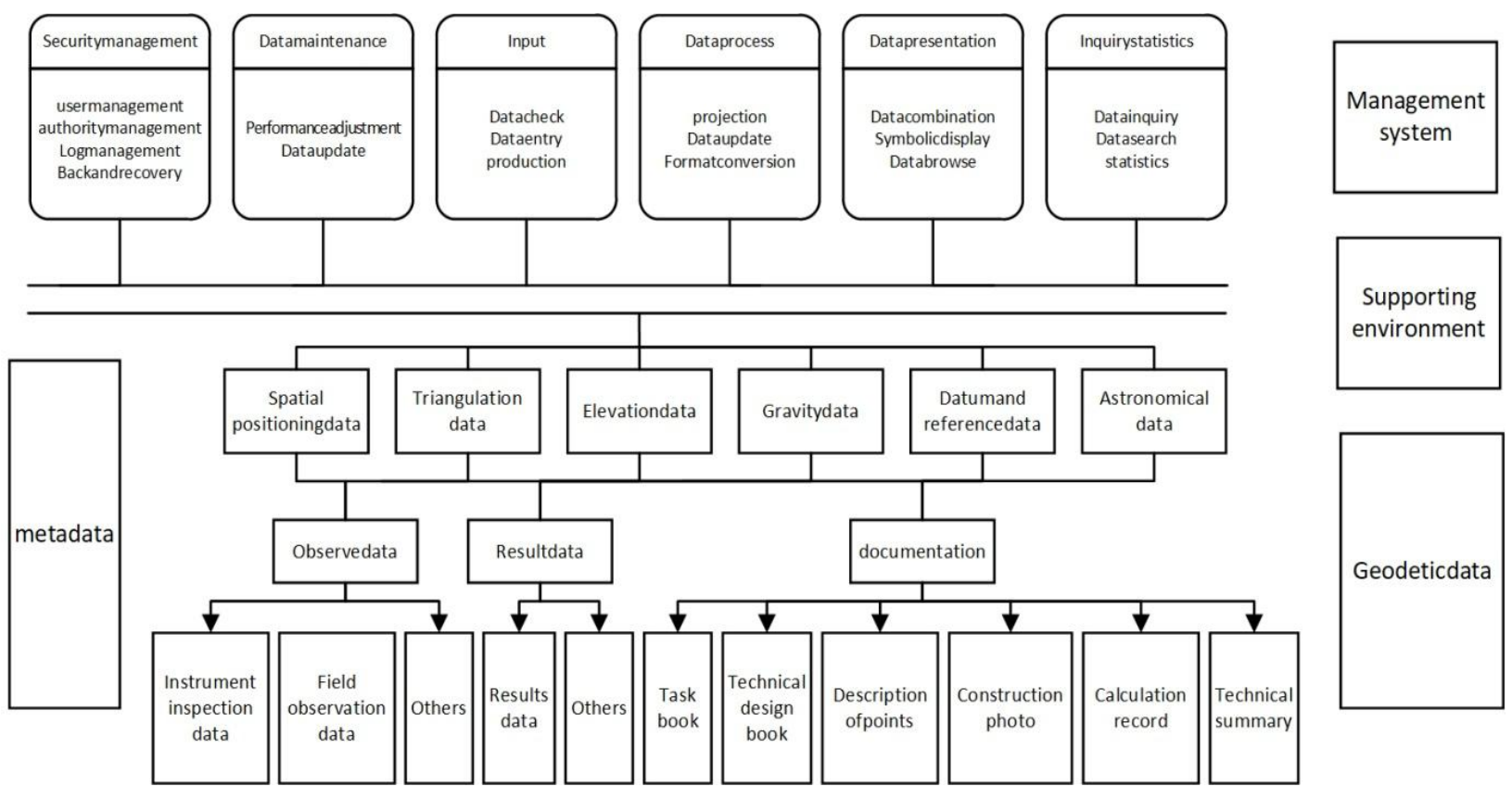

Figure 4, Geodetic Database System

\section{CONCLUSION}

The standardization of data resulting from the production and application of digital geodetic measurements has attracted more and more attention in recent years. People realize that without data standards, data sharing services are impossible. This paper studies the data content, characteristics, classification principles and classification methods in the modern geodetic technology system and initially forms a standard classification system of geodetic data. On the basis of studying the key technical links of modern geodetic data acquisition, storage, management and service, this paper has tackled the real-time data optimal storage based on DBFS technology.

The technology reflected by the standard classification system proposed in this paper is the key to realize the geodetic data sharing service environment. On the one hand, they are used as relatively independent technical data in geodetic operations, geodynamics research, and operational geodetic support; on the other hand, they are horizontally combined with other professional information standards in the field of surveying and mapping into spatial information standard systems, which are applied to space information science and its applications and services.

\section{REFERENCES}

State Bureau of Quality and Technical Supervision. GB 22021-2008 Basic Technical Provisions for National Geodesy [S]. Beijing. China Standard Publishing House, 2008: 2-4

Lei Zhang, Main functions and services of National Geodetic Data Base [J]. Geographic Information World. 2003, (2): 26-29
National Bureau of Quality and Technical Supervision. Basic Requirements for Geodetic Data Base of GH/T 2012-2013 [S]. Beijing. China Standards Publishing House, 2013: 3-5

Party Yamin, Mijin zhong, Yingyan Cheng, Principle and Application of Navigation Satellite System [M]. Beijing: Surveying and Mapping Publishing House, 2007:52-54

Jingnan Liu, Zhiping Lu. Establishment of Geodetic Data Sharing Environment [J]. Surveying and Mapping Bulletin, 2003, (4): 1-4

Dongqing Zhao, Zhiping Lu. Research on Geodetic Web Service Framework and Its Application [J]. Surveying and Spatial Geographic Information, 2008, 33 (4): 203-205

Yongshang Wang, Xiaohua Wang, Xiaoqing Wang, et al. Geodetic data classification research and construction [J]. Surveying and mapping science, 2014, 39 (12): 24-28

Xiangyuan Kong, Jiming Guo, Zongquan Liu, Basis of Geodesy [M]. Wuhan: Wuhan University Press, 2005: 367-368

Libo Yao, Renli Wang, GIS data management based on Oracle Spatial database $[\mathrm{J}]$. Surveying, mapping and spatial geographic information, 2006, 29 (2): 81-83

Junyong Chen, Wen Hanjiang, Cheng Pengfei, Some problems in the development of Geodesy in China [J]. Journal of Wuhan University (Information Science Edition), 2001, 26 (6): 475-482

Fengjuan Wu. Dynamic Establishment of Geodetic Data Model [J]. Marine Surveying and Mapping, 2006, (4), 39-41 
The International Archives of the Photogrammetry, Remote Sensing and Spatial Information Sciences, Volume XLII-3/W10, 2020 International Conference on Geomatics in the Big Data Era (ICGBD), 15-17 November 2019, Guilin, Guangxi, China

Aimin Xu, Zhiping Lu, Xuerui Li, etc. Application of XML in Geodetic Data Sharing Environment [J]. Journal of College of Surveying and Mapping, 2004, 21 (02): 87-89

Jingnan Liu, Zhiping Lu. Study on the Standard System of Geodetic Data [J]. Surveying and Mapping Bulletin, 2003, (8):1-3

Yongshang Wang, Network Construction of National Geodetic Database [J]. Journal of Surveying and Mapping, 2003

(Supplement), 34 (2): 40-44 